\title{
Comparative bronchial vasoconstrictive efficacy of inhaled glucocorticosteroids
}

\author{
E.S. Mendes*, A. Pereira*, I. Danta*, R.C. Duncan\#, A. Wanner*
}

Comparative bronchial vasoconstrictive efficacy of inhaled glucocorticosteroids. E.S. Mendes, A. Pereira, I. Danta, R.C. Duncan, A. Wanner. C ERS Journals Ltd 2003.

ABSTRACT: The vasoconstrictive efficacies of glucocorticosteroids (GS) are usually compared by the McKenzie skin-blanching test and taken as an index of relative potency. The rationale for the present study was to transpose the McKenzie test to the airway and to compare the airway vascular effects of three inhaled GS: beclomethasone dipropionate (BDP), fluticasone propionate (FP) and budesonide (BUD), in healthy subjects and patients with mild stable asthma.

A soluble, inert gas-uptake method was used to measure airway blood flow ( $\left.Q_{\text {aw }}\right)_{i}$ Baseline mean \pm SD $Q_{\text {aw }}$ normalised for anatomical dead space was $53.1 \pm 1.4 \mu \mathrm{L} \cdot \mathrm{min}^{-1} \cdot \mathrm{mL}^{-1}$ in healthy subjects $(\mathrm{n}=10)$ and $67.8 \pm 3 \mu \mathrm{L} \cdot \mathrm{min}^{-1} \cdot \mathrm{mL}^{-1}$ in asthmatics $(\mathrm{n}=10)$.

All GS caused a transient decrease in $Q_{\text {aw. }}$ The magnitude of the vasoconstriction was greater in asthmatics. The relative vasoconstrictive effect of BDP, FP and BUD was $1,1.9$, and 2.7, respectively, in asthmatics and $1,3.3$ and 3.0, respectively, in healthy subjects, as assessed by the dose required to decrease $Q$ aw by $20 \%$ from the baseline, 30-min postdrug inhalation.

Therefore, measuring airway blood flow may be a useful, site-specific parameter to assess the tissue bioavailability and vasoconstrictive efficacy of inhaled glucocorticosteroids. Eur Respir J 2003; 21: 989-993.
*Pulmonary and Critical Care Division, Dept of Medicine, University of Miami at Mount Sinai and ${ }^{\#}$ Dept of Epidemiology and Public Health, University of Miami, Miami, FL, USA.

Correspondence: A. Wanner, Division of Pulmonary and Critical Care Medicine, University of Miami School of Medicine, P.O. Box 016960 (R-47), Miami, FL 33101, USA. Fax: 13052436992

E-mail: awanner@miami.edu

Keywords: Asthma bronchial blood flow corticosteroid vasoconstriction

Received: August 82002

Accepted after revision: February 102003

This research was supported by an academic research grant from Glaxo Inc., Middlesex, UK.
Inhaled glucocorticosteroids (GS) have assumed an important role in the treatment of asthma. Various outcome measures have been used to quantitate the clinical efficacy (maximum effect) of inhaled GS [1-4]. However, efficacy cannot be equated with potency (receptor-binding affinity). However, both efficacy and potency are important in establishing a therapeutic index. The in vivo "potency" (tissue bioavailability and vasoconstrictive efficacy) of inhaled GS is commonly determined by the McKenzie skin-blanching test [5]. The test is based on the ability of inhaled GS to cause transient cutaneous vasoconstriction when applied topically in healthy subjects. However, for inhaled GS, the McKenzie skin-blanching test is not ideal because the test is performed in the skin as a substitute for the airway, it is time-consuming and involves normal rather than inflamed tissue. The latter is considered a shortcoming as airway inflammation has been shown to alter the responsiveness to vasoactive agents [6]. Airway tissue is the therapeutic target for inhaled GS. Therefore, the airway can be considered the ideal site to assess the tissue bioavailability and vasoconstrictive efficacy of inhaled GS.

The authors have developed and validated an in vivo technique for the measurement of airway blood flow ( $Q$ aw) in humans [7]. They have shown that $Q$ aw is increased in asthmatics, presumably due to inflammatory new vessel formation and vasodilatation [6, 7]. They also found that inhaled fluticasone propionate (FP) causes a dose-dependent, transient decrease in $Q$ aw in healthy and asthmatic subjects, with a greater response observed in the latter [8,9]. These findings indicate that $Q$ aw is a suitable index of tissue bioavailability and vasoconstrictive efficacy for inhaled GS.

The purpose of the present study was to compare the vasoconstrictive effect of three commercially available inhaled GS preparations: FP, budesonide (BUD) and beclomethasone dipropionate (BDP), in healthy and asthmatic subjects.

\section{Methods}

\section{Test subjects}

A total of 10 subjects with mild asthma and 10 normal volunteers, without any history of asthma or other respiratory disease, were recruited for this study. All subjects were current nonsmokers. Asthma was defined by American Thoracic Society criteria [10]. At study entry, all asthmatics were clinically stable and had a forced expiratory volume in one second $(\mathrm{FEV} 1)>70 \%$ predicted, rare daytime symptoms and no nocturnal awakenings (mild intermittent asthma [11]). The asthmatics had not used inhaled or systemic GS or regularly administered $\beta$-adrenergic agonists for a minimum of 2 weeks before the study.

None of the subjects were taking oral anti-inflammatory agents or vasoactive medications, or had cardiovascular disease. All subjects denied having experienced an acute respiratory infection $<1$ month before the study and no subject developed an acute respiratory infection during the study.

The study protocol was approved by the Mount Sinai Medical Center and the University of Miami Institutional Review Boards. All subjects provided written, informed consent. They received financial remuneration for their participation. 


\section{Forced expiratory volume in one second}

Spirometry was carried out using an Essential Medic Unit (model 6200 Autobox DL; Yorba Linda, CA, USA). The FEV1 was determined and expressed as an absolute value and as $\%$ pred [12].

\section{Airway blood flow}

A previously validated and applied, soluble, inert gasuptake was used to measure $Q$ aw [7, 13]. The subjects first inhaled room air and then exhaled $500 \mathrm{~mL}$ from the total lung capacity position. Subsequently, the subjects rapidly inhaled the same volume of gas from a gas mixture contained in a Teflon bag, consisting of 10\% dimethylether (DME), 5\% helium and a balance of oxygen. After a predetermined breath-hold time, the subject then exhaled into a spirometer, through a critical flow orifice, to standardise the expiratory flow. During exhalation, the instantaneous concentrations of DME, nitrogen and helium were measured at the airway opening using a mass spectrometer (Perkin-Elmer, Pomona, CA, USA) along with the expired gas volume. The manoeuvre was performed with two breath-hold times each of 5, 10, 15 and $20 \mathrm{~s}$ in random order. The $Q$ aw was calculated by multiplying the helium-corrected DME concentration slope by the expired anatomic dead space volume (minus the proximal $50 \mathrm{~mL}$ ) to obtain DME uptake, which was then divided by the mean DME concentration and the solubility coefficient for DME in blood (Fick's principle). The anatomic dead space was derived from the nitrogen washout curve inscribed after the 10-s breath-hold time. $Q$ aw was expressed as $\mu \mathrm{L} \cdot \mathrm{min}^{-1} \cdot \mathrm{mL}^{-1}$ anatomical dead space.

\section{Protocol}

The subjects were instructed to abstain from ingesting alcoholic beverages the night before the study and the asthmatic subjects were asked not to use their inhaled $\beta$-adrenergic agonist for $\geqslant 12 \mathrm{~h}$ before the study. For each subject, the experiment was started at the same time on the different study days. The subjects were asked not to ingest coffee or caffeinated drinks prior to the study.

Days 1-3: time course. The subjects inhaled either BDP $(1,680 \mu \mathrm{g}), \mathrm{FP}(880 \mu \mathrm{g})$ or BUD $(1,000 \mu \mathrm{g})$ from commercially available metered-dose inhalers (MDIs) with a spacer in random order on three different days. The manoeuvre was standardised by having the subjects inhale from a functional, residual-capacity position to a total lung-capacity position, followed by a 10 -s breath-hold. The $Q$ aw and FEV1, blood pressure and pulse rate were measured before and at 15-, 30-, 60-, 90- and 120-min postdrug inhalation.

Days 4-6: dose/response. On each of these 3 days, the subjects underwent a dose/response assessment of one of the three GS preparations in random order. The doses were as follows: 420 , $840,1,680$ and $3,360 \mu \mathrm{g}$ for BDP; $220,440,880$ and $1,760 \mu \mathrm{g}$ for FP; and 200, 400, 800 and 1,600 $\mu \mathrm{g}$ for BUD. After the baseline measurements of blood pressure, pulse rate, $Q$ aw and FEV1, the lowest dose was inhaled and the measurements repeated $30 \mathrm{~min}$ later. This corresponded to the nadir of $Q$ aw after inhalation of FP in a previous study [9]. Immediately thereafter, the next highest dose was inhaled and the procedure repeated until the maximum dose had been inhaled.

\section{Statistical analysis}

The unpaired and paired variants of t-tests were used to compare baseline data among and within the two groups. A univariate and multivariate, repeated-measures analysis were used to analyse and compare the time courses to the three drugs. To assess the dose/response, the appropriate statistical model was used, analysing the response within each subject and pooling across subjects, using the method of dummy variables to adjust for different levels of response across subjects.

The response profiles were modelled within subjects as:

$$
Q_{\text {aw }} \mathrm{i}=\mathrm{a}_{\mathrm{i}} \mathrm{e}^{- \text {bDose }}
$$

where $\mathrm{i}$ is the subject number, a the intercept, $\mathrm{b}$ the decay constant and e the base of natural log, using a natural log transform to $\ln (Q$ aw $\mathrm{i})=\ln \left(\mathrm{a}_{\mathrm{i}}\right)-$ bDose. Use of the dummy variables allowed the computation of a common decay constant $\mathrm{b}$. The final model was:

$$
Q_{\mathrm{aw}}=\mathrm{ae}^{-\mathrm{bDose}}
$$

where a is back-calculated from the average of the baseline $\ln (Q \mathrm{aw})$ across subjects. The adequacy of this procedure was assessed by the $\mathrm{R}^{2}$ value associated with the final model.

Once the model was computed for each drug and each group, the per cent reduction from baseline was calculated to find an appropriate point within the range of the data to estimate relative vasoconstrictive efficacy. For a given per cent of baseline $(\mathrm{P})$, the dose necessary to reach that point was calculated as:

$$
\operatorname{Dose}_{\mathrm{p}}=-\ln (\mathrm{P})^{-1}
$$

Relative vasoconstrictive efficacy was calculated as the ratio of the respective Dose $\mathrm{p}_{\mathrm{p}}$ values. The SD was derived according to the principle of the propagation of error. Data were expressed as mean \pm SD. A $\mathrm{p}<0.05$ was considered significant.

\section{Results}

Demographic and baseline physiological data for the two study groups are shown in table 1 . The mean Qaw was $29 \%$ higher in subjects with asthma when compared with healthy subjects $(\mathrm{p}<0.001)$. The mean FEV1 was lower in subjects with asthma compared with the healthy subjects $(\mathrm{p}<0.001)$. All other parameters were similar in the two groups of subjects. There were no statistically significant variations in baseline $Q$ aw among experimental days (fig. 1). Likewise, mean baseline blood pressure, pulse rate, and FEV1 remained

\begin{tabular}{|c|c|c|}
\hline & Healthy & Asthmatics \\
\hline Subjects $n$ & 10 & 10 \\
\hline Age yrs & $31(18-41)$ & $38(28-52)$ \\
\hline Male $\mathrm{n}$ & 3 & 2 \\
\hline Female $\mathrm{n}$ & 7 & 8 \\
\hline Weight kg & $67.1 \pm 4.9$ & $65.3 \pm 3.4$ \\
\hline Heart rate $\cdot \min ^{-1}$ & $70 \pm 1$ & $70 \pm 1$ \\
\hline Systolic BP mmHg & $110 \pm 1$ & $111 \pm 2$ \\
\hline Diastolic BP mmHg & $71 \pm 1$ & $71 \pm 1$ \\
\hline FEV1 L & $3.41 \pm 0.07$ & $2.70 \pm 0.10^{*}$ \\
\hline FEV1 \% pred & $92.9 \pm 1.1$ & $86.8 \pm 1.5^{*}$ \\
\hline$Q$ aw $\mu \mathrm{L} \cdot \mathrm{min}^{-1} \cdot \mathrm{mL}^{-1}$ & $53.1 \pm 1.4$ & $67.8 \pm 3 *$ \\
\hline Anatomic dead space mL & $202 \pm 8$ & $178 \pm 5$ \\
\hline
\end{tabular}
stable throughout the study.

Table 1.-Demographics and baseline physiological parameters

Data are presented as mean \pm SD on 6 experiment days unless otherwise stated. BP: blood pressure; FEV1: forced expiratory volume in one second; $Q$ aw: airway blood flow. *: $\mathrm{p}<0.05$. 


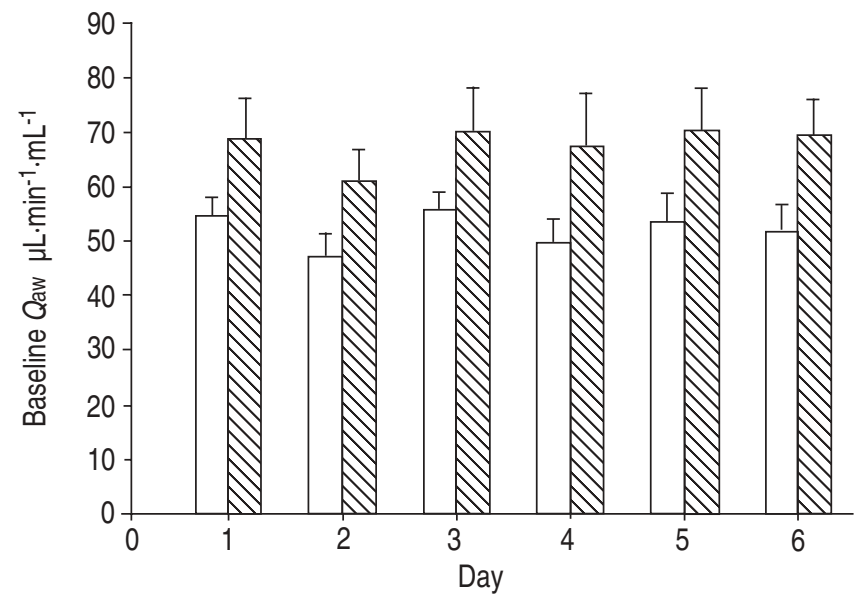

Fig. 1.-Baseline airway blood flow ( $\left.Q_{\text {aw }}\right)$ in healthy ( $\square$ ) and asthmatic subjects $(\mathbb{\otimes})$. Data are presented as mean \pm SD.

There were no changes in mean FEV1, anatomical dead space, pulse rate or blood pressure after inhalation of any of the three GS in either asthmatic or healthy subjects.

No adverse effects were reported after drug inhalation. Some subjects experienced transient sleepiness that was attributed to DME.

\section{Time course of glucocorticosteroid effect on airway blood flow}

All subjects showed a marked, statistically significant decrease in Qaw after inhalation of BDP, FP and BUD (figs 2 and 3). The nadir in mean $Q$ aw was observed at 60 min in all instances except for BUD in healthy subjects, where the nadir occurred at $90 \mathrm{~min}$. As the time of maximal decrease in Qaw showed interindividual variability, the authors calculated the maximum change in $Q$ aw for each subject. The mean maximum decrease was greater in asthmatics compared with healthy subjects for all three GS examined ( $\mathrm{p}<0.05$; table 2$)$.

\section{Dose-dependent effects of glucocorticosteroid effect on airway blood flow}

There was a dose/response relationship for all three drugs in both groups of subjects (figs 4 and 5). The relative potency of BDP, FP and BUD was 1, 1.9 and 2.7, respectively, in asthmatic subjects and $1,3.3,3.0$, respectively, in healthy subjects,

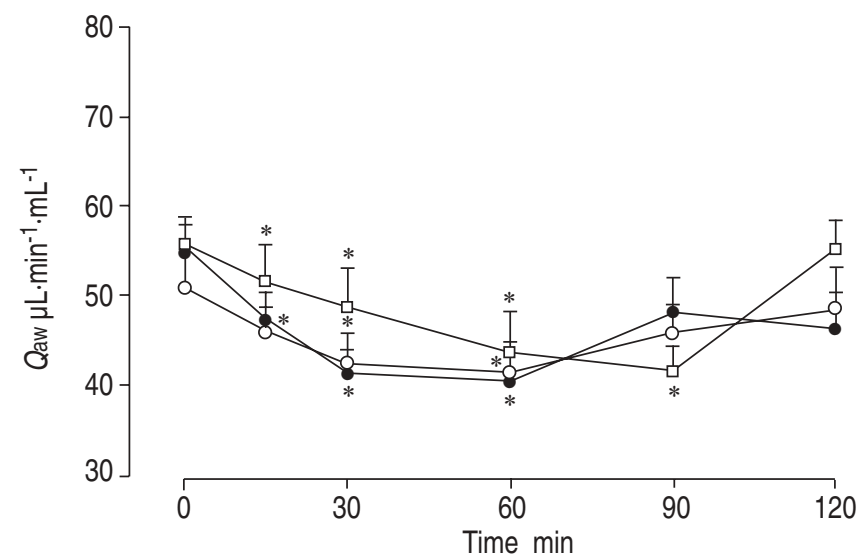

Fig. 2.-Airway blood flow $\left(Q_{\text {aw }}\right)$ before and after the inhalation of fluticasone $(\bigcirc)$, beclomethasone $(\bigcirc)$ and budesonide $(\square)$ in healthy subjects $(\mathrm{n}=10)$. Data are presented as mean \pm SD. *: $\mathrm{p}<0.05$ versus baseline value.

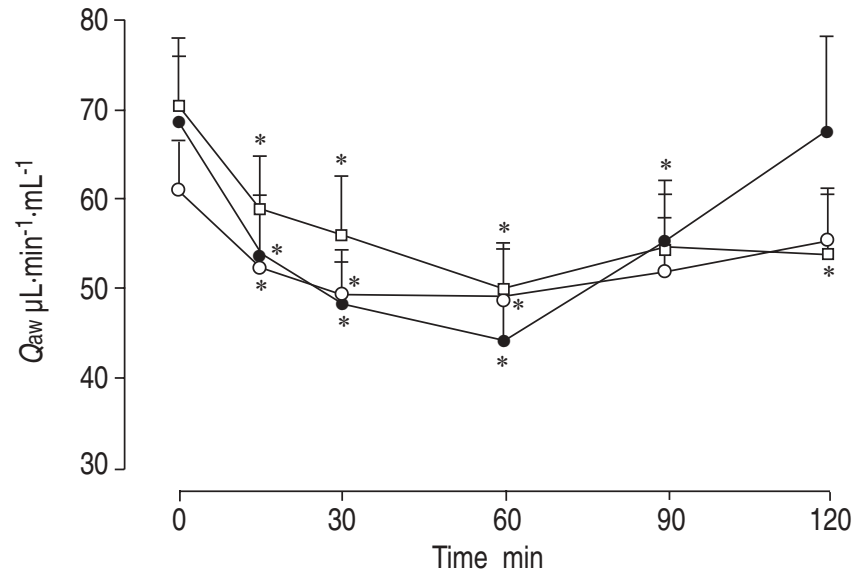

Fig. 3. - Airway blood flow ( $\left.Q_{\text {aw }}\right)$ before and after the inhalation of fluticasone $(\mathbf{O})$, beclomethasone $(\bigcirc)$ and budesonide $(\square)$ in asthmatic subjects $(\mathrm{n}=10)$. Data are presented as mean \pm SD. *: $\mathrm{p}<0.05$ versus baseline value.

as assessed by the dose required to decrease $Q$ aw by $20 \%$ from the baseline $(\mathrm{p}<0.05$ for FP and BUD versus BDP; table 3$)$.

\section{Discussion}

Although inhaled GS are commonly used in the treatment of asthma, the relationship between dose and clinical response remains unclear. A number of inhaled GS have been

Table 2. - The maximum decreases in airway blood flow (Qaw) $\mu \mathrm{L} \cdot \mathrm{min}^{-1} \cdot \mathrm{mL}^{-1}$ after fluticasone propionate (FP), beclomethasone dipropionate (BDP) and budesonide (BUD) inhalation in healthy and asthmatic subjects

\begin{tabular}{lccc}
\hline & \multicolumn{3}{c}{ Glucocorticosteroids } \\
\cline { 2 - 4 } & FP & BDP & BUD \\
\hline Concentration $\mu \mathrm{g}$ & 880 & 1680 & 1000 \\
Healthy $^{\#}$ & $21.8 \pm 2.0^{*}$ & $15.3 \pm 2.7^{*}$ & $23.3 \pm 3.1^{*}$ \\
Asthmatics & $30.05 \pm 4.9$ & $21.1 \pm 3.4$ & $29.7 \pm 4.4$
\end{tabular}

Data are presented as mean $\pm \mathrm{SD}$ of individual troughs in the timecourse protocol. \#: $\mathrm{n}=10 ; *: \mathrm{p}<0.05$ versus corresponding value in asthmatics.

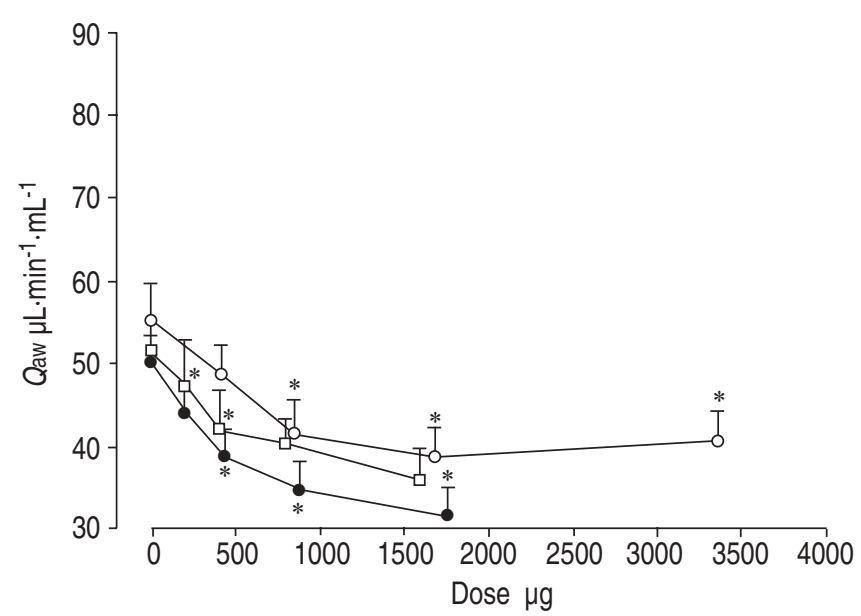

Fig. 4. - Dose/response relationship between inhaled fluticasone $(\bullet)$ beclomethasone $(\bigcirc)$ and budesonide $(\square)$ doses and airway blood flow $\left(Q_{\text {aw }}\right)$ in healthy subjects $(\mathrm{n}=10)$. Data are presented as mean \pm SD. *: $\mathrm{p}<0.05$ versus baseline value. 


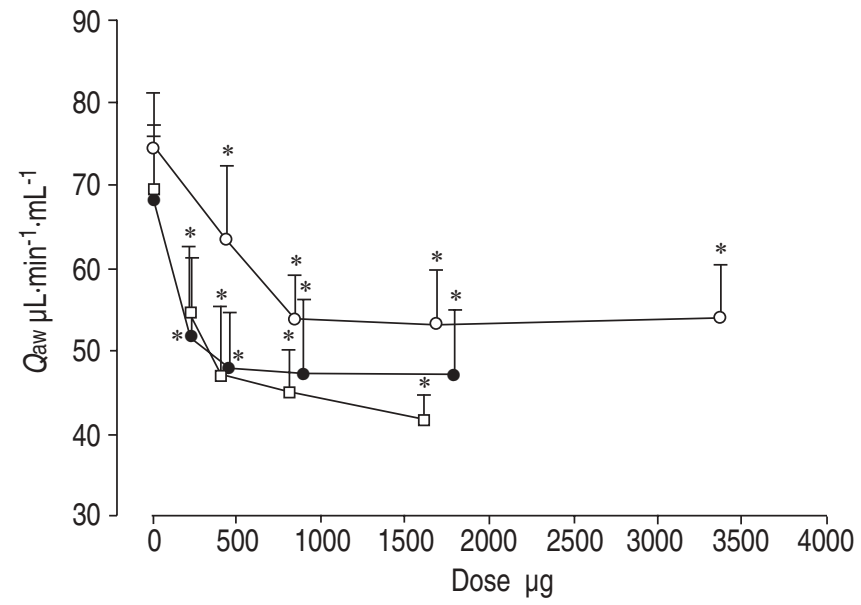

Fig. 5.-Dose/response relationship between inhaled fluticasone ( $)$, beclomethasone $(\bigcirc)$ and budesonide $(\square)$ dose and airway blood flow $\left(Q_{\text {aw }}\right)$ in asthmatics $(\mathrm{n}=10)$. Data are presented as mean \pm SD. *: $\mathrm{p}<0.05$ versus baseline value.

introduced over the years, with different pharmacokinetics, pharmakodynamics, potencies and bioavailabilities and more efficient delivery systems have been developed. [2, 14, 15]. Differences in pharmacokinetics determine the topical to systemic effect ratio or the pulmonary targeting of the drug. Differences in receptor-binding affinity, however, translate into differences in potency for different drugs.

There is no method to evaluate the potency of inhaled GS in vivo. The standard screening test to determine the relative "potencies" for inhaled GS has been the McKenzie skinblanching test [5]. However, this procedure has come under criticism. The intensity of the blanching response to a GS varies from subject-to-subject and is influenced by ambient temperature and humidity and other factors [16]. The blanching effect score is difficult to compare among groups or among specific formulations conducted in different groups and the visual reading of the skin-blanching by the human eye is a subjective measure. Furthermore, the results are influenced by drug diffusibility through tissue, which is likely to vary in skin and in the airway. Most importantly, the test assesses vasoconstrictive efficacy rather than true potency (receptor-binding affinity) and may have little relevance to the anti-inflammatory effects of GS, which, in contrast to vasoconstriction, are mediated through genomic effects. Finally, aerosol delivery devices can produce clinically significant differences in topical activity by altering the dose deposited in the lung [17]. Alternative approaches are therefore desirable.

The authors believe that the quantitative assessment of bronchial vasoconstriction, as described in this article, is such

Table 3.-Relative vasoconstrictive efficacy of beclomethasone dipropionate (BDP), fluticasone propionate (FP) and budesonide (BUD) in healthy $(n=10)$ and asthmatic subjects $(n=10)$

\begin{tabular}{lccccc}
\hline & \multicolumn{2}{c}{ Healthy } & & \multicolumn{2}{c}{ Asthmatics } \\
\cline { 2 - 3 } \cline { 5 - 6 } & EC20 $\mu \mathrm{g}$ & Relative efficacy & & EC20 $\mu \mathrm{g}$ & Relative efficacy \\
\hline BDP & $2721 \pm 863$ & 1 & & $2625 \pm 988$ & 1 \\
FP & $823 \pm 155^{*}$ & 3.3 & & $1336 \pm 416^{*}$ & 1.9 \\
BUD & $922 \pm 183^{*}$ & 3.0 & & $970 \pm 337^{*}$ & 2.7 \\
\hline
\end{tabular}

Data are presented as mean \pm SD unless otherwise stated. EC20: effective concentration that causes a $20 \%$ decrease in airway blood flow from baseline. *: $\mathrm{p}<0.05$ versus beclomethasone. an alternative approach. The vascular response is measured at the anatomically relevant site for inhaled GS, using a clinically relevant inhalation technique and with the inclusion of clinically relevant test subjects, such as asthmatics.

Inhalation technique, particle size distribution and airway geometry all influence aerosol deposition in the airways. Although the inhalation manoeuvre was normalised and a spacer was used, MDI-generated, particle-size distribution may have differed for the three drugs used. Asthmatic and healthy subjects have different airway geometry, which, in turn, influences total aerosol deposition and deposition distribution. These variables are part of tissue bioavailability and may significantly influence the authors' vasoconstrictive efficacy measurement for the three MDIs used clinically.

The change of $Q$ aw in response to inhaled GS is a physiological parameter that reflects bronchial vascular tone because inhaled GS have no effect on bronchial arterial perfusion (aortic) and presumably downstream (atrial) pressures. Therefore, there is a direct relationship between vascular conductance (which is indirectly related to bronchial vascular smooth muscle tone) and $Q$ aw. It is unlikely that $Q$ aw was influenced by the manoeuvres involved in this study's protocol, based on previous observations with a chlorofluorocarbon placebo MDI [9].

The dose/response measurements were taken at 30-min intervals because the authors had previously demonstrated that FP at $880 \mu \mathrm{g}$ produced a transient decrease in mean $Q$ aw with a nadir at $30 \mathrm{~min}$ in asthmatic and healthy subjects [9] and because it was the first time point where all three drugs showed a significant change in $Q$ aw in healthy and asthmatic subjects. Analysis of the dose/response curve at 60 min may have changed the relative vasoconstrictive efficacy ratio for the three drugs.

The relative vasoconstrictive efficacies of BDP, FP and BUD were 1, 1.9 and 2.7, respectively, in asthmatic subjects and $1,3.3$ and 3.0, respectively, in healthy subjects. Using the McKenzie skin-blanching test, KAMADA et al [18] found the vasoconstrictive efficacy of inhaled GS relative to dexamethasone to be 600 for BDP, 980 for BUD and 1,200 for FP [18]. While different relative efficacies have been reported by other investigators, the rank order of $\mathrm{BDP}<\mathrm{BUD}<\mathrm{FP}$ has been consistent across laboratories [19, 20]. The discrepancies may result from differences in the technique of applying the drug to the skin or the subjective assessment of skin blanching. The interdrug quantitative differences between the present observations and the reported skin-blanching findings may be related to the differences in tissue bioavailability.

The mechanism of the rapid transient vasoconstrictor response to topically applied GS is unknown. Preliminary findings suggest that $\alpha$-adrenergic neurotransmission is involved [21]. The concentration of noradrenaline (NA) at $\alpha_{1}$-adrenergic receptor sites is, in part, regulated by NA uptake into postsynaptic cells (extraneural uptake: uptake ${ }_{2}$ ) where NA undergoes enzymatic inactivation. It has been demonstrated in vitro that, uptake ${ }_{2}$ is inhibited by steroid hormones through a nongenomic action [22]. This could lead to an increased NA concentration at the neuromuscular junction and explain the GS-induced vasoconstriction. The potentiated GS-induced vasoconstriction in asthmatics' airways seems to be accompanied by a greater $\alpha_{1}$-adrenergic vasoconstrictor response [6]. This adds further support to an $\alpha$-adrenergic-GS interplay in the regulation of vascular tone.

The $Q$ aw response to inhaled GS is an in vivo index of airway tissue bioavailability and vasoconstrictive efficacy, not true potency or clinical anti-inflammatory efficacy. Although the vasoconstrictive differences reported here, for three popular inhaled GS, may not affect their clinical efficacy, the authors believe it is essential to consider tissue bioavailability when clinically evaluating GS. The Global Initiative for Ashma 
guidelines, recommend dosages for different inhaled GS on a $\mu \mathrm{g} \cdot \mathrm{day}^{-1}$ basis, implying that no differences exist among the products [23]. However, both in vitro and in vivo evaluations, including the present study, have demonstrated interdrug differences in potency and bioavailability [18-20]. Such data are needed to establish a therapeutic index $[20,24]$.

In summary, this investigation showed that inhaled fluticasone propionate and budesonide cause greater vasoconstriction in the airway than beclomethasone dipropionate. It was also shown that for all three inhaled glucocorticosteroids, the vasoconstrictor response is greater in asthmatics than in healthy subjects. These findings indicate drug-specific and disease-specific in vivo potency differences in both bioavailability and vasoconstrictive efficacy among three commonly prescribed glucocorticosteroid metered-dose inhalers.

\section{References}

1. Martin RJ, Szefler SJ, Chinchilli, et al. Systemic effect comparisons of six inhaled corticosteroid preparations. Am J Respir Crit Care Med 2002; 165: 1377-1383.

2. Nielsen LP, Dahl R. Therapeutic ratio of inhaled corticosteroids in adult asthma. Am J Respir Crit Care Med 2000; 162: 2053-2057.

3. Barnes PJ. Inhaled glucocorticosteroids for asthma. $N$ Engl J Med 1995; 332: 868-875.

4. Djukanovic R, Wilson JW, Britten K, et al. Effect of an inhaled corticosteroid on airway inflammation and symptoms in asthma. Am Rev Respir Dis 1992; 145: 669-674.

5. McKenzie AW, Stoughton RB. Method for comparing percutaneous absorption of steroids. Arch Dermatol 1962; 86: 608-610.

6. Brieva J, Wanner A. Adrenergic airway vascular smooth muscle responsiveness in healthy and asthmatic subjects. J Appl Physiol 2001; 90: 665-669.

7. Kumar SD, Emery MJ, Atkins ND, Danta I, Wanner A. Airway mucosal blood flow in bronchial asthma. Am J Respir Crit Care Med 1998; 158: 153-156.

8. Brieva JL, Danta I, Wanner A. Effect of an inhaled glucocorticosteroid on airway mucosal blood flow in mild asthma. Am J Respir Crit Care Med 2001; 161: 293-296.

9. Kumar SD, Brieva JL, Danta I, Wanner A. Transient effect of inhaled fluticasone on airway mucosal blood flow in subjects with and without asthma. Am J Respir Crit Care Med 2000; 161: 918-921.

10. American Thoracic Society. Guidelines for the diagnosis and treatment of patients with chronic pulmonary disease (COPD) and asthma. Am Rev Respir Dis 1987; 136: 225-244.
11. National Heart, Lung and Blood Institute and National Asthma Education Program. Guidelines for the diagnosis and management of asthma. J Allerg Clin Immunol 1991; 88: 425-534.

12. Crapo RO, Morris AH, Gardner RM. Reference spirometric values using techniques and equipment that meet ATS recommendation. Am Rev Respir Dis 1981; 123: 659-664.

13. Scuri M, McCaskill V, Chediak AD, Abraham WM, Wanner A. Measurement of airway mucosal blood flow with dimethyl-ether: validation with microspheres. $J$ Appl Phisiol 1995; 79: 1386-1390.

14. Bousquet J, Ben-Joseph R, Messonnier M, Alemao E, Gould AL. A meta-analysis of the dose response relationship of inhaled corticosteroids in adolescents and adults with mild to moderate persistent asthma. Clin Ther 2002; 42: 1-20.

15. Holt S, Suder A, Weatherall M, Cheng S, Shirtcliffe P, Beasley R. Dose-response relation of inhaled fluticasone propionate in adolescents and adults with asthma: metaanalysis. $B M J$ 2001; 323: 1-8.

16. Vinod PS, Peck CC, Skelly JP. Vasoconstriction skinblanching assay for glucocorticoids - a critique. Arch Dermatol 1989; 125: 1558-1561.

17. Wilson AM, Dempsey OJ, Coutie WRJ, Sims E, Lipworth BJ. Importance of drug-device interaction in determining systemic effects of inhaled corticosteroids. Lancet 1999; 353: 2128.

18. Kamada A, Szefler SJ, Martin RJ, Boushey HA, Chinchilli VM, Drazen JM. Issues in the use of inhaled corticosteroids. Am J Respir Crit Care Med 1996; 153: 1739-1748.

19. Barnes PJ, Pedersen S. Efficacy and safety of inhaled corticosteroids in asthma. Am Rev Respir Dis 1993; 148: S1-S26.

20. Kelly HW. Establishing a therapeutic index for the inhaled corticosteroids: Pharmacokinetic/pharmacodynamic comparison of inhaled corticosteroids. J Allergy Clin Immunol 1998; 102: S36-S51.

21. Martel F, Azevedo I, Oswald W. Extraneural uptake and O-methylation of $3 \mathrm{H}$-adrenaline in rabbit aorta. Naunyn Scmiedebergs Arch Pharmacol 1993; 347: 363-370.

22. Horvath G, Lieb T, Conner GE, Salathe M, Wanner A. Steroid sensitivity of norepinephrine uptake by human bronchial arterial and rabbit aortic smooth muscle cells. Am J Respir Cell Mol Biol 2001; 25: 500-506.

23. Global Initiative for Asthma. Global strategy for asthma management and prevention NHLBI/WHO workshop report. Publication no. 95-3659. Bethesda, National Institutes of Health, National Heart, Lung and Blood Institute, 1995.

24. Kelly WH. Comparison of inhaled corticosteroids. Ann Pharmacother 1998; 32: 220-232. 